\title{
Three Hospitals in China
}

\author{
Robin A. Haig, Consultant Psychiatrist, Royal Hobart Hospital, Tasmania
}

In April 1983 I went on a medical tour of China, travelling around the People's Republic in three weeks, with compulsory stops at the Friendship Stores, and flying visits to commune hospitals, clinics, a Chinese Medicine hospital, and a Chinese Medicine factory. The psychiatrists on the tour were interested in visits to the Shanghai Psychiatric Hospital, the Neuropsychiatric Department of the Beijing Capital Hospital and the Hangzhau Railway Workers' Sanitarium.

I was interested in discovering how the psychiatrically ill are treated in a population of more than 1000 million, with only 3500 psychiatrists and 60,000 psychiatric beds, as estimated by Walls et al (1975). This represents approximately one psychiatrist per 250,000 population, which compares with one psychiatrist per 1 million population in India, as estimated by Wig (1982), and one psychiatrist (consultant or SHMO) per 36,000 population in the United Kingdom, which emerges from the Report of the College Manpower Working Party (Royal College of Psychiatrists, 1982). In China, Taiwan and Sichuan, reports suggest that the incidence rates of schizophrenia and manic depressive illness are comparable to those found in population studies from other cultures (Lin and Kleinman, 1981; Lin, 1982; Liu, 1980).

\section{The Shanghai Psychiatric Hospital}

This is the psychiatric hospital which appears to be the most commonly visited by foreign visitors to China (Brown, 1980). Shanghai was previously a cosmopolitan city, although purged of its multitudinous vices after 1949. Many people on the street speak English, and are more accustomed to foreigners than in many other parts of China. The hospital is well organized, presentable and close to the city centre, with helpful staff, who have become used to showing foreign visitors around. There is a permanent interpreter on the staff. The hospital is a clean, modern, but basic series of buildings, dating from 1958, with extensions still being constructed.

The Shanghai Psychiatric Hospital is a 'municipal hospital'. In addition there are district (or county) hospitals (of 60 to 100 beds), with one mental hospital per district. There are also commune or urban clinics which seem to cater for cases away from the centre of the cities which do not require (or do not receive) specialized treatment.

The Shanghai Psychiatric Hospital is a 'municipal distributed amongst 15 wards (one being an isolation ward for patients with infectious diseases). Males are segregated from females on the wards and all patients wear uniforms. The out-patient department handles 450 patients daily. There are occupational therapy and recreational therapy departments. Included on the staff are 178 doctors, 350 nurses and $\mathbf{4 0}$ technicians. Investigative facilities consist of a pathology laboratory, EEG, and psychological testing (the WAIS, MMPI and Rorschach are all utilized). There are said to be departments of genetics, statistics, epidemiology and psychopathology.

The ICD9 is used for classification of psychiatric illnesses. The majority of in-patients are diagnosed as schizophrenic. In 1968,85 per cent of in-patients were schizophrenic, but in 1982, the reported proportion decreased to 75 per cent. Over a 20-year period, only 64 alcoholics were admitted for treatment, and these were mainly foreign sailors in transit. General paralysis of the insane is now said to be nonexistent, following the campaign for eradication of venereal diseases after 1949. Sexual problems do not present to psychiatrists. (Our guides were slightly puzzled over the emphasis given to sexual problems in the West.)

A typical severe depressive illness (in Shanghai) consists of marked sadness, withdrawal and guilt feelings, the patient sometimes having delusions that he has harmed his family, with frequent suicidal ideas. Five per cent of patients with an affective disorder seen by the hospital subsequently commit suicide. Common paranoid delusions (in schizophrenic illness) include delusions of persecution (rarely involving the state or political system) and delusions of control by electricity, rays, instruments or computers. There are very few religious delusions reported. Patients with pathological jealousy sometimes present.

There does not appear to be a legal form which ensures that the patient who is ill is treated when he is refusing help. Patients are said always to agree to hospital admission when it is advised by the doctor. They may come to hospital voluntarily, or may be escorted by a family member or member of the street committee.

Some treatments (e.g. electroconvulsive treatment) were not available at certain hospitals during the Cultural Revolution. ECT is now utilized widely in the treatment of depressive illness, catatonia and schizophrenia. This is administered in the bilateral, unmodified mode, except in the elderly, where a muscle relaxant and anaesthetic are employed. Ten treatments, given over three to four weeks, constitute an 'average' course of ECT.

Tricyclic antidepressants are used in depressed patients and some phobic patients. The dose range of amitriptyline is $150 \mathrm{mgs}$ to $250 \mathrm{mgs}$ daily, given in three divided doses. Intravenous clomipramine has been used in patients with obsessional illnesses. Major tranquillizers used in schizophrenia include trifluoperazine, chlorpromazine, perphenazine, haloperidol, clozapine and fluphenazine enanthate. In acute psychosis, a total daily chlorpromazine dose of $300 \mathrm{mgs}$ to $500 \mathrm{mgs}$ is used. Intramuscular fluphenazine enanthate, $25 \mathrm{mgs}$ to $50 \mathrm{mgs}$ weekly, is given to schizophrenics. Lithium carbonate is used in treating 
patients with manic illness.

Psychotherapy consists of an educational approach, to correct incorrect thoughts (parallel to Rational Emotive Therapy). In group therapy there is a discussion of the behaviour and thinking of the individual, in the context of current Chinese political and social policies. However, this latter therapy may not be a specifically psychiatric treatment, as it occurs widely in the population in general. (Counselling and psychotherapy of many conditions, including neurosis and personality disorder, which in the West are conducted by a professional person, are conducted in China by street committees, party members, fellow workers or the family.) Social pressures to conform to acceptable standards of behaviour exist everywhere in the world, but may be more formally organized in China than elsewhere. A 'group leader' is appointed by the staff from amongst the patients, and is usually a patient who has almost recovered, is more vocal, and can help to guide the other patients in the right direction. Follow-up of outpatients is preferably performed by the same doctor or health worker, although the concept of 'transference' does not appear to be recognized as such. Behaviour therapy is apparently used in certain neurotic conditions. The occupational therapy department is involved in making dolls, tablecloths, and other small items, profits from the sale of these being used for hospital facilities. Recreational therapy consists of games, such as ping-pong, music and dancing, and Tai Ji (traditional slow motion exercises).

Acupuncture is used (in combination with other treatments) for treating a wide range of psychiatric symptoms. Specific acupuncture points are used for certain symptoms and illnesses, e.g. a point in the tragus of the ear is useful in decreasing auditory hallucinations, and a point in the sole of the foot is used in treating stupor. It is claimed that acupuncture controls the side effects of phenothiazines. Administration of acupuncture in the Shanghai Psychiatric Hospital is described by Leung and Miller (1978). Chinese herbal medicine is employed in a wide range of conditions, usually in combination with other treatments. It is used as the only physical treatment in minimal brain damage in children. The underlying theoretical basis of Chinese Medicine (i.e. traditional medicine) is founded on a body of theory originating in the 12th Century B.C., and includes concepts of Yin and Yang, Tao, the five fundamental elements, the seasons and weather, spirits and demons, emotions, trauma, hunger, overeating and wrong methods of meditation (Koran, 1972). Multifactorial causation of illness is a tradition of. Chinese Medicine. Dualistic concepts of mind and body were never paramount in Chinese Medicine. In some respects there are parallels with modern psychiatric thinking, with notions of multifactorial causation of illness, and psychosomatic integration, which make it easier for traditional and modern theories of psychiatry to co-exist. Classification, diagnosis and treatment of psychiatric conditions in traditional Chinese Medicine are described by Liu (1980). Traditional
Chinese herbal medicines have recently been tested in psychiatric conditions in this hospital, in the treatment of a special periodic psychosis, occurring premenstrually, of apathy in schizophrenics, and of psychosis following carbon monoxide poisoning. The treatment of schizophrenics in Sichuan, with extracts from the herbs Parasiticus on Coriaria and its host, Coriaria, was described by Liu (1980). The active ingredients of these two herbs are Tutin and Coriamyrtin, which provoke a seizure, but the side effects limit their usefulness.

There are now attempts to meet the shortages in manpower by extending training schemes. Each year 500 medical students receive psychiatric training in this hospital. In the fourth year of undergraduate training there are 36 hours of psychiatric lectures, and a two-week clinical attachment to the psychiatric hospital in the fifth year. Postgraduate training to become a psychiatrist occurs over three years whilst working in this hospital. The lecture course comprises clinical psychiatry in the first year, differential diagnosis and neurology in the second year, and "biopsychiatry' (including genetics, biochemistry, psychological testing and physiology) in the third year-exams are held at the end of each year of the course. Psychiatrists are trained in the use of acupuncture and Chinese herbal medicines as well as in the use of modern psychotropic compounds, ECT and insulin shock treatment. Schools of influence include Pavlov, Kraepelin and Adolf Meyer; polite interest is expressed in Freud, although his ideas are not altogether acceptable in terms of Marxism-Leninism.

Supplies of textbooks and journals are very limited and are eagerly devoured by doctors working in these hospitals. A Chinese textbook of psychiatry (published in 1980) was unobtainable from booksellers in Shanghai-sold out. Some articles on psychiatry are published in the Chinese Medical Journal. The hospital received a limited number of overseas journals, including the American Journal of Psychiatry, the British Journal of Psychiatry, Acta Psychiatrica Scandinavica and Archives of General Psychiatry. Great interest was expressed by our guides when the possibility of providing further psychiatric journals and literature was suggested. In general, there appears to be a determination by the staff we met to raise standards, to train more personnel and to share knowledge frmm other countries. Postgraduate experience in psychiatry and neurology in hospitals in the West would be eagerly welcomed by trainees and experienced doctors from China.

\section{The Beifing Capital Hospital}

We were fortunate to be able to visit this hospital and meet the Professor of Neuropsychiatry, Prof. Y. K. Feng. This teaching hospital was founded in 1921 with the aid of the Rockefeller Foundation and has 650 beds, with departments in all specialties, plus a Chinese Medicine Department. The staff of 1900 include 100 technical personnel, 450 doctors (including 100 professors and assistant professors) 
and 150 nurses. Sixty nurses and 30 medical students per year qualify from the hospital.

The eight-year medical school curriculum includes two and a half years' preclinical studies, two and a half years' clinical sciences (including physiology, biochemistry and anatomy) and three years' attachment to hospital departments. During the Cultural Revolution, the medical school was closed (a fate shared by many other academic establishments), and morale, progress, research and teaching all suffered major setbacks. However, the morale of the staff is now improving, new equipmert is being obtained (a CT scanner and a linear scanner included), and professors and students are sometimes able to travel to overseas centres and conferences.

The Department of Neuropsychiatry has $\mathbf{4 0}$ beds in the hospital. The staff consist of 25 doctors (including two professors and three assistant professors) and 32 nurses. Psychiatry and neurology have not been divorced as specialties in China, so the specialists we met regarded themselves as neuropsychiatrists. Nursing shortages are common, caused partly by fiscal restraint and partly by the generous maternity leave. Two-thirds of the beds are occupied by patients with cerebrovascular disease, who present a placement problem, as there are not always relatives available to care for the disabled stroke victim. A rehabilitation department and a 'family follow-up service', with a domiciliary nurse attached, have been established to assist with these problems. The other in-patients in this Department have a wide variety of neurological conditions. Patients with a psychiatric illness who require hospitalization are transferred to the local psychiatric hospital, where the Department has 60 beds. There are busy emergency clinics (seeing 2000 patients annually), liaison services (seeing 600 patients annually) and out-patient clinics (seeing 150 patients daily). There are also out-patient clinics for private patients and high ranking officials. (There is a limited right of private practice amongst some doctors in China.)

Research interests of the department include calcium metabolism in Wilson's Disease, EMG studies in the detection of childhood diabetes, EEG studies of infantile spasm, the incidence of multiple sclerosis and an innovative technique from performing sphenoidal lead EEGs utilizing very fine acupuncture needles, devised by Feng et al (1983).

In the opinion of Professor Feng, major tranquillizers and ECT are more effective than herbal remedies or acupuncture in the treatment of psychoses. Acupuncture treatment of schizophrenia involves a needle being inserted in the 'Feng Fu' point, which is just above the first cervical vertebra and can potentially cause transection of the cord. In rural areas, the barefoot doctors, who receive limited medical training. are able to diagnose psychosis, but are not sufficiently skilled to diagnose subtypes, so ECT is used as a universal treatment for psychosis. It is given initially on a twice daily basis for three days, and then as necessary. This approach produces a rapid relief of symptoms in many psychotic patients and enables them to rapidly resume their work in the commune.or factory. This treatment is usually performed in the commune hospital, as the municipal hospitals are remote, and the peasant and his family cannot afford the fare to the city.

\section{Hangzhau Railway Workers' Sanitarium}

We were able to observe the treatment of certain neurotic illnesses and psychosomatic conditions in this hospital. It is a hospital of 250 beds, established 30 years ago, for treating patients with non-infectious chronic diseases, including cardiovascular, respiratory, locomotor, psychiatric and occupational diseases. Multiple treatments are utilized, including physiotherapy, Chinese herbal medicines, acupuncture, moxibustion, dietary treatments, physical exercises (including Tai Ji) and 'milieu therapy' (e.g. teaching people how to live a regular life, making use of the beautiful scenery, increasing their general health education and generally raising their morale). The treatment of 'neurosis' includes the above list and, in addition, a type of low voltage electrical stimulation which is applied to the temples and forehead for 20 minutes daily for 15 days in 'category I neurosis', whereas, in 'category II neurosis' the patient sits on a low voltage electrical pad for 15 minutes daily for 15 days. This treatment effectively charges the patient up with electricity. If he recovers with this treatment, it may be repeated. Category I neurosis is a 'disease of the mind, accompanied by insomnia, dizziness and excitement' and category II neurosis presents with 'pains in the arms and legs'. Hypnotics are rarely employed in the treatment of insomnia.

\section{Psychosocial ruminations}

Whilst modern theories and systems of psychiatric classification and treatment are being used in the larger hospitals, it is apparent that these co-exist alongside traditional approaches. The Chinese social system, with its emphasis on society rather than the individual, and the traditional Chinese family values, both play a part in the development, presentation and management of psychiatric illness. There is still stigma attached to psychiatric illness in the traditional Chinese family. However, there appears to be a tolerance towards psychiatric illness by many state enterprises, which accept patients back again, providing they are not overtly disturbed, nor hampering productivity. Social rehabilitation may be aided by the cohesive links between family or commune members. Theories of psychiatric illness in China include notions that the mental activities of man are a reflection of the objective world in the brain. Correct attitudes towards illness, future life, work and study (particularly study of dialectical materialism) are all thought to help the patient recover more rapidly and prevent relapse. In contrast to the findings of Brown (1980), the teachings of Mao are no longer being stressed as the panacea. Pavlov's theories, inherited from the days of the Russian alliance, still 
hold considerable respect. Freudian and developmental theory are being considered, although the individualistic, intrapsychic emphasis of psychoanalysis, psychoanalytic theories on the origins of aggression, and emphasis on libido and sexuality do not appear to be too readily acceptable. Psychology as an academic discipline was regarded as a non-productive and mildly subversive activity during the Cultural Revolution, although it is now being reinstated. Chinese child care facilities would appear to cast doubt on the theories of many eminent Western psychologists. Boarding kindergartens exist for children aged three to six years, in which the children only visit parents at weekends, do not possess their own toys (or transitional objects) and appear to grow up into well adjusted citizens.

In some ways, China could be regarded as a massive social laboratory, in which, following the excesses and disorganization of the Cultural Revolution, regulated social changes, many of a pragmatic nature, are being implemented. The outcome, in terms of psychosocial pathology (or lack of it) will be observed over the next generation.

ACKNOWLEDGEMENTS

I would like to thank Dr. Sheng Han Xu and Dr. Jun Mian Xu of Shanghai Psychiatric Hospital, 600 Wang Ping Nan Road, Shanghai, China, and Prof. Y. K. Feng, of Department of Neuropsychiatry, The Capital Hospital Beijing, China, for their guidance.

REFERENCES

Brown, L. B. (1980) A psychologist's perspective on psychiatry in
China. Australian and New Zealand Journal of Psychiatry. 14, 21-35.

FenG, Y. K., Xu, J. Q. \& Guo, D. H. (1983) The use of acupuncture needles as sphenoidal electrodes in electroencephalography: Observation of 2000 cases. Chinese Medical Journal, 96, 211-18.

KORAN, L. (1972) Psychiatry in mainland China: history and recent status. A merican Journal of Psychiatry, 128, 970-78.

LeUNG, M. R. S. \& MilleR, M. H. (1978) Chinese approach to mental health service. Canadian Psychiatric Association Journal, 23, 354-60.

Liv, X. (1980) Mental health work in Sichuan. British Journal of Psychiatry, 137, 371-76.

LIN. T. (1982) Culture and psychiatry: A Chinese perspective. Australian and New Zealand Journal of Psychiatry, 16, $235-45$.

LiN, K. M. \& KLenman, A. (1981) Recent development of psychiatric epidemiology in China. Culture, Medicine and Psychiatry, 5, 135-43.

Royal College of Psychiatrists (1982) Report of the Manpower Working Party: Medical Manpower in the Psychiatric Specialties. Bulletin of the Royal College of Psychiatrists, 6, 18-24.

Walls, P. D., Walls, L. H. \& Langsley, D. G. (1975) Psychiatric training and practice in the People's Republic of China. American Journal of Psychiatry, 132, 121-28.

Wig, N. N. (1982) Psychiatric research in India. Bulletin of the Royal College of Psychiatrists, 6, 102-104.

Xu. S. H. (1982) Traditional Chinese medicine in mental illness. Chinese Medical Journal, 93, 325-28.

\section{A Psychiatric Version of the New Aberdeen Medical Record}

Kerth J. B. Rix, Lecturer in Psychiatry, University of Manchester (now Senior Lecturer in Psychiatry, University of Leeds); BetTy McNally, Psychiatric Records Officer, University Hospital of South Manchester; and Margaret Johnson, former Psychiatric Unit Administrator, University Hospital of South Manchester

When Wilson et al (1978) described 'The new Aberdeen Medical Record,' they stated: 'The bulk and disarray of many hospital medical records make it impossible to review and retrieve information easily, hamper the proper care of patients and cause much time to be wasted ... when papers accumulate chaotically in the folders much time is wasted in clumsy attempts to retrieve information; important facts about patients and their problems and drug treatment are obscured.' The same problems apply to the records of psychiatric patients, and this paper describes the adaptation of the Aberdeen Medical Record for use in psychiatric units and hospitals.

\section{Description of the new paychiatric record}

The principle of the record is the same as that of the Aberdeen record in that 'like' documents are grouped together. Whereas the Aberdeen record has four sections, the psychiatric record has six sections: group A-patient identification sheets; group B-correspondence and summaries; group C-written case records; group D-reports; group E-investigation reports; group F-miscellaneous. These six sections are separated by five coloured cardboard dividers with protruding tabs. The tabs are labelled 'Correspondence', 'Case records', 'Reports', 'Investigations' and 'Miscellaneous'.

The first divider has printed on it a list of the six groups for filing purposes, followed by the filing instructions for group $A$ and group B. The second to fifth dividers have printed on them the filing instructions for groups $C$ to $F$ respectively.

Group A: This group, comprising in-patient (HMR 1 (PSYCHI.I/P)) and out-patient identification sheets (HMR 1 